Preprint typeset in JHEP style - HYPER VERSION

FSU-HEP-020408

OHSTPY-HEP-T-02-004

\title{
Viable models with non-universal gaugino mediated supersymmetry breaking
}

\author{
Howard Baer, Csaba Balázs, Alexander Belyaev* \\ Department of Physics, Florida State University \\ Tallahassee, FL, USA 32306 \\ E-mail: baer@hep.fsu.edu, balazs@hep.fsu.edu, belyaev@hep.fsu.edu \\ Radovan Dermíšek \\ Department of Physics, The Ohio State University, \\ Columbus, $\mathrm{OH}$ 43210, USA \\ E-mail: dermisek@pacific.mps.ohio-state.edu \\ Arash Mafi \\ Department of Physics, University of Arizona \\ Tucson, AZ 85712 \\ E-mail: mafi@physics.arizona.edu \\ Azar Mustafayev \\ Department of Physics, Florida State University \\ Tallahassee, FL, USA 32306 \\ E-mail: mazar@hep.fsu.edu
}

ABSTRACT: Recently, extra dimensional SUSY GUT models have been proposed in which compactification of the extra dimension(s) leads to a breakdown of the gauge symmetry and/or supersymmetry. We examine a particular class of higher-dimensional models exhibiting supersymmetry and $S U(5)$ or $S O(10)$ GUT symmetry. SUSY breaking occurs on a hidden brane, and is communicated to the visible brane via gaugino mediation. Nonuniversal gaugino masses are developed at the compactification scale as a consequence of a restricted gauge symmetry on the hidden brane. In this case, the compactification scale is at or slightly below the GUT scale. We examine the parameter space of such models where gaugino masses are related due to a Pati-Salam symmetry on the hidden brane. We find limited but significant regions of model parameter space where a viable spectra of SUSY matter is generated. Our results are extended to the more general case of three independent gaugino masses; here we find that large parameter space regions open up for large values of the $U(1)$ gaugino mass $M_{1}$. We also find the relic density of neutralinos for these models to be generally below expectations from cosmological observations, thus leaving room for hidden sector states to make up the bulk of cold dark matter. Finally, we evaluate the branching fraction $B F(b \rightarrow s \gamma)$ and muon anomalous magnetic moment $a_{\mu}$. 
KEYwords: Supersymmetry Breaking, Supersymmetry Phenomenology, GUT.

\footnotetext{
* On leave of absence from Nuclear Physics Institute, Moscow State University.
} 


\section{Introduction}

Introducing supersymmetry (SUSY) to solve the hierarchy problem gives rise to a puzzle of identifying the way it is broken. Several mechanisms for SUSY breaking have been invented. Although at present there is no compelling reason to prefer one to the other, we know that the soft SUSY breaking terms in the minimal supersymmetric standard model (MSSM) must have a very special form. In order to avoid unacceptably large flavor violation, the masses of squarks and sleptons must be degenerate to a high precision ${ }^{1}$. Similarly the trilinear couplings must be proportional to the Yukawa matrices. Therefore it is intriguing to explore scenarios which naturally produce soft SUSY breaking parameters with these desired properties. Examples of such scenarios are gauge mediation [1], anomaly mediation [2, 3], and gaugino mediation [4, 5].

In the gaugino mediation scenario, the MSSM matter fields are localized on a 3-brane - the "matter" brane embedded in higher dimensional spacetime - while gauge fields can propagate in the bulk of the extra dimensions. Supersymmetry is broken on a separate "hidden sector" brane which is separated from the matter brane in extra dimensions. Gauginos can directly couple to the source of SUSY breaking to obtain nonzero masses, while masses of squarks and sleptons and trilinear couplings are suppressed due to the spatial separation of the branes. Thus, just below the compactification scale $M_{c}^{2}$, the effective 4-dimensional theory is the MSSM and in the minimal version gaugino masses are the only non-negligible soft SUSY breaking parameters. ${ }^{3}$ Scalar masses and trilinear couplings receive large contributions from gaugino masses through the renormalization group (RG) running between $M_{c}$ and the electroweak (EW) scale. These contributions are flavor blind and therefore the resulting soft SUSY breaking terms at the EW scale cause only a modest flavor violation coming from the Yukawa couplings.

In earlier works on gaugino mediation [4, 5], the compactification scale was assumed to be at or above the GUT scale, in order to preserve the success of gauge coupling unification. Therefore all gaugino masses are equal at $M_{c}$. For $M_{c}=M_{G U T}$, however, this scenario predicts the lightest stau $\tilde{\tau}_{1}$ to be the lightest supersymmetric particle (LSP), which violates cosmological bounds on the existence of stable charged or colored relics from the Big Bang in models which conserve $R$-parity. The situation is different for $M_{c}>M_{G U T}$. In this case the additional RG running between $M_{c}$ and $M_{G U T}$ has to be considered [5]. This generates non-zero scalar masses and trilinear couplings at the GUT scale; most importantly the stau mass gets a positive contribution which eventually can make the $\tilde{\tau}_{1}$ heavier than the lightest neutralino $\widetilde{Z}_{1}$. This removes the unpleasant charged LSP feature of the scenario with $M_{c}=M_{G U T}\left[\right.$ [5]. In models which assume a simple GUT beyond $M_{G U T}$, the values of $\tan \beta(\sim 35-50)$ consistent with Yukawa coupling unification do not match the $\tan \beta$ values $(\sim 10-25)$ predicted by the presence of a small bilinear soft SUSY breaking parameter $B_{0}$ [6].

\footnotetext{
${ }^{1}$ Other solutions to the SUSY flavor problem such as decoupling or alignment are generally more awkward to implement in models.

${ }^{2} M_{c} \sim 1 / R, \mathrm{R}$ represents the size of extra dimension(s).

${ }^{3}$ Soft SUSY breaking Higgs masses can also be generated if Higgs fields propagate in the bulk.
} 
There has been recent interest in constructing GUT models in higher dimensional spacetimes [17, 8, 9, 10, 11]. Orbifold compactification of the extra dimensions is an elegant way of achieving GUT symmetry breaking and the doublet triplet splitting of Higgs fields. Proton decay due to dimension 5 operators that is a serious problem in 4-dimensional GUT models [12], can be naturally suppressed in these models. The common feature of these models is the existence of a brane or several branes at orbifold fixed points on which the gauge symmetry is restricted to be a subgroup of the GUT symmetry. This results in an effective 4-dimensional theory with gauge symmetry given by an intersection of gauge symmetries on orbifold fixed points.

Positive features of GUTs, like charge quantization or gauge coupling unification, can be preserved. If matter multiplets live in the bulk or are localized on a brane which respects the GUT symmetry, then they come in complete GUT multiplets. Furthermore, if the compactification scale is not far from $M_{G U T}$, the gauge couplings still unify. The gauge couplings run from the EW scale to the compactification scale with the ordinary MSSM $\beta$ functions. They almost unify at $M_{c}$ which is below but very close to the conventional GUT scale. Beyond $M_{c}$, the gauge couplings run according to the power-law rule [13] due to the heavy KK modes that do not fill degenerate GUT multiplets; they ultimately unify at a scale $M_{*}$, the cutoff scale of the model.

The existence of a brane with restricted gauge symmetry can play an important role for gaugino mediation. If SUSY is broken on a brane with restricted gauge symmetry, nonuniversal gaugino masses are generated. For example, if using proper boundary conditions $S U(5)$ is broken on a brane down to the standard model (SM), non-universal gaugino masses $M_{1}, M_{2}, M_{3}$ can be generated on this brane [8].

Even more interesting is the situation for $S O(10)$ models in higher dimensions [10, 11] which can contain branes with gauge symmetries being different subgroups of $S O(10)$. Using proper boundary conditions, fixed branes with Pati-Salam $S U(4) \times S U(2)_{L} \times S U(2)_{R}$, Georgi-Glashow $S U(5) \times U(1)$, or flipped $S U(5)^{\prime} \times U(1)^{\prime}$ gauge symmetries can be obtained. If gauginos get masses on these branes, the gauge symmetry relates gaugino masses of the MSSM at the compactification scale. In the case of Pati-Salam gauge symmetry, gaugino masses $M_{2}$ and $M_{3}$ are free parameters while the $M_{1}$ is given by a linear combination of $M_{2}$ and $M_{3} 10$.

The compactification scale in these models is below the GUT scale and the boundary conditions with negligible squark and slepton masses and trilinear couplings are realized at this scale. Therefore the cure of Ref. [5] to the stau LSP problem doesn't apply here. However, in this case the non-universal gaugino masses may help to obtain viable SUSY spectra with a neutralino LSP, at least in some restricted regions of model parameter space. Non-universal gaugino masses can also be very helpful in order to obtain Yukawa coupling unification for models with a positive $\mu$ term[14].

We emphasize that extra dimensional gauge theories are not the only way to get the MSSM at the GUT scale with the non-universal gaugino masses as the only sources of the soft SUSY breaking. For example, one may imagine deconstructing the 5-dimensional models with non-universal gaugino masses (for discussion of deconstructed universal gaugino mediation see [15]). In addition, models of no-scale supergravity can also provide us 
with the desired boundary conditions i.e. vanishing scalar masses and trilinear couplings and non-zero gaugino masses [16]. In a no-scale locally supersymmetric GUT, a non-trivial choice of the couplings between the vector supermultiplets and the chiral supermultiplet can easily result in non-universal gaugino masses at the GUT scale [17]. Similar results can also be obtained in type I string constructions, see for example [18].

In this paper, we present the allowed region of SUSY parameter space and study the supersymmetric particle spectrum for the case where the bino mass $M_{1}$ is related to $M_{2}$ and $M_{3}$ by the Pati-Salam symmetry. In this case we $d o$ find regions of parameter space leading to a viable SUSY particle spectrum although the allowed regions turn out to be rather tightly restricted. We also evaluate the relic density of neutralinos for these models; we find it in general to be somewhat below expectations from cosmological measurements due to the presence of a non-negligible higgsino component to the LSP. This is a positive feature of such models, since one may expect additional cold dark matter (CDM) states associated for instance with the hidden brane. We evaluate as well the branching fraction $B F(b \rightarrow s \gamma)$ and muon anomalous magnetic moment $a_{\mu}=\frac{(g-2)_{\mu}}{2}$. We also study the effect of adding non-zero soft SUSY breaking Higgs masses on the allowed regions of parameter space; typically, these Higgs masses do not ameliorate the situation. Finally, we examine the case of completely independent non-universal gaugino masses at the compactification scale. In this case, we find that regions of parameter space leading to viable spectra open up for large values of the bino mass $M_{1}$. We show sample spectra for these models; they typically lead to spectra with nearly degenerate $\widetilde{W}_{1}$ and $\widetilde{Z}_{1}$, since the LSP turns out to be either wino-like (as in AMSB models) or higgsino-like.

This paper is organized as follow. In Sec. 2, we review some details of extra dimensional SUSY GUT models with gaugino mediated SUSY breaking, and their associated parameter space. In Sec. 3, we examine models where $M_{1}$ is determined in terms of $M_{2}$ and $M_{3}$ by the Pati-Salam gauge symmetry, and show that there exists parameter space leading to viable models, although its extent is somewhat limited. In Sec. \$, we present our results for completely independent gaugino masses. These models emphasize regions of parameter space with large $U(1)_{Y}$ gaugino mass $M_{1}$, and can frequently lead to spectra with a winolike LSP. Our conclusions are presented in Sec. 5 .

\section{Non-universal Gaugino Mediation}

In this section, we will review the basic features of GUT models in higher dimensional spacetimes. We start with the simple example of an $S U(5)$ GUT in five dimensions (for more detailed discussion, see [8]). The fifth dimension is compactified on an $S^{1} /\left(Z_{2} \times Z_{2}^{\prime}\right)$ orbifold where $S^{1}$ is a circle of radius $\mathrm{R}$ defined with a periodic coordinate $0 \leq y<2 \pi R$. $Z_{2}$ identifies opposite points on the circle $y \rightarrow-y$ and $Z_{2}^{\prime}$ identifies opposite points with respect to $y^{\prime}=y-\pi R / 2$, i.e. $y^{\prime} \rightarrow-y^{\prime}$. The resulting orbifold has two inequivalent fixed points $O$ at $y=0$ and $O^{\prime}$ at $y=\pi R / 2\left(y^{\prime}=0\right)$. There are fixed branes (4dimensional Minkowski spaces) at these points. Let's assume that the gauge supermultiplet

and two Higgs hypermultiplets in $\mathbf{5}$ and $\overline{\mathbf{5}}$ representations propagate in the bulk. In five dimensions, $\mathcal{N}=1$ supersymmetry is generated by 8 supercharges and is equivalent to 
$\mathcal{N}=2$ supersymmetry in four dimensions. Using 4 dimensional superfield notation, the 5-dimensional gauge supermultiplet can be written as $(V, \Sigma)$ where $V$ is a 4-dimensional gauge supermultiplet and $\Sigma$ is a chiral supermultiplet, both in the adjoint (24-dimensional) representations of $S U(5)$. Similarly Higgs hypermultiplets that transform as $\mathbf{5}$ and $\overline{\mathbf{5}}$ can be written as $\left(H_{5}, H_{5}^{c}\right)$ and $\left(H_{\overline{5}}, H_{\overline{5}}^{c}\right)$ where $H_{5}$ and $H_{5}^{c}\left(H_{\overline{5}}\right.$ and $\left.H_{\overline{5}}^{c}\right)$ are 4-dimensional chiral supermultiplets in the $\mathbf{5}$ and $\overline{\mathbf{5}}(\overline{\mathbf{5}}$ and $\mathbf{5})$ representations of $S U(5)$.

A generic field $\phi\left(x_{\mu}, y\right)$ in the 5-dimensional bulk is identified by its transformations under the $Z_{2}$ and $Z_{2}^{\prime}$ parities $P= \pm$ and $P^{\prime}= \pm$, respectively; $\phi(-y)=P \phi(y)$ and $\phi\left(-y^{\prime}\right)=P^{\prime} \phi\left(y^{\prime}\right)$. Only those fields with parity $(+,+)$ under $Z_{2} \times Z_{2}^{\prime}$ have massless Kaluza-Klein modes in the resulting 4-dimensional effective theory. Invariance of the 5dimensional gauge interactions under $Z_{2}$ requires a relative minus sign between the parity transformations of $V$ and $\Sigma$ and similarly between $H$ and $H^{c}$ (in both $\mathbf{5}$ and $\overline{\mathbf{5}}$ ). $Z_{2}$ parities of $V, H_{5}$ and $H_{\overline{5}}$ are taken to be + and those of $\Sigma, H_{5}^{c}$ and $H_{\overline{5}}^{c}$ are taken to be -. Therefore the orbifold compactification breaks $\mathcal{N}=2$ supersymmetry in four dimensions down to $\mathcal{N}=1$.

Using a non-trivial assignment of $Z_{2}^{\prime}$ parities $P^{\prime}=\operatorname{diag}(-1,-1,-1,+1,+1)$ acting on 5, the $\mathrm{SU}(5)$ gauge symmetry is restricted to the standard model gauge symmetry $S U(3)_{C} \times S U(2)_{L} \times U(1)_{Y} \equiv G_{S M}$ on the $O^{\prime}$ brane. At the same time $P^{\prime}$ splits Higgs doublets from their color triplet partners. As a result only gauge fields of the minimal supersymmetric standard model and two Higgs doublets $H_{u}$ and $H_{d}$ (those contained in $H_{5}$ and $\left.H_{\overline{5}}\right)$ are $(+,+)$ fields and therefore will be present at the massless level in the resulting 4-dimensional effective theory.

The third generation matter fields can be localized on the $O$ brane. Since the gauge symmetry on the $O$ brane is $\mathrm{SU}(5)$, matter fields must come in complete $\mathrm{SU}(5)$ multiplets. Therefore we can have usual $\mathrm{SU}(5) b-\tau$ Yukawa unification although the 4-dimensional theory is just the MSSM.

This setup with two separated fixed branes is very suitable for gaugino mediation. Let's assume that SUSY is broken by the vacuum expectation value of a gauge singlet chiral superfield $X$ that is localized on the $O^{\prime}$ brane,

$$
\langle X\rangle=\theta^{2} F_{X}
$$

$X$ can couple directly to the gauge fields on the $O^{\prime}$ brane through the ultraviolet scale suppressed terms

$$
\mathcal{L}_{O^{\prime}} \supset \int d^{2} \theta\left(\lambda_{3} \frac{X}{M_{*}^{2}} W^{i \alpha} W_{\alpha}^{i}+\lambda_{2} \frac{X}{M_{*}^{2}} W^{j \alpha} W_{\alpha}^{j}+\lambda_{1} \frac{X}{M_{*}^{2}} W_{Y}^{\alpha} W_{Y \alpha}+H . c .\right),
$$

where index $i(j)$ runs over the gauge fields of the $S U(3)(S U(2))$ gauge group. This will give masses to gauginos of $S U(3), S U(2)_{L}$ and $U(1)_{Y}$ separately,

$$
M_{i}=\frac{\lambda_{i} F_{X} M_{c}}{M_{*}^{2}}, \quad i=1,2,3
$$

which corresponds to the fact that the gauge symmetry on the $O^{\prime}$ brane is restricted to $G_{S M}$. The factor $M_{c}$ (the compactification scale, $M_{c} \sim 1 / R$ ) comes from the wave function 
normalization of the 4-dimensional gaugino fields. Similarly the $\mu$ and $B_{\mu}$ terms $\left(B_{\mu}=B \mu\right)$ can be generated:

$$
\mathcal{L}_{O^{\prime}} \supset \int d^{4} \theta\left(\frac{X^{\dagger}}{M_{*}^{2}} H_{u} H_{d}+\frac{X X^{\dagger}}{M_{*}^{3}} H_{u} H_{d}+H . c .\right)
$$

leading to

$$
\mu \sim \frac{F_{X} M_{c}}{M_{*}^{2}}, \quad B_{\mu} \sim \frac{F_{X}^{2} M_{c}}{M_{*}^{3}} .
$$

Finally soft SUSY breaking masses for Higgs scalars can be generated:

$$
\mathcal{L}_{O^{\prime}} \supset \int d^{4} \theta\left(\frac{X X^{\dagger}}{M_{*}^{3}} H_{u} H_{u}^{\dagger}+\frac{X X^{\dagger}}{M_{*}^{3}} H_{d} H_{d}^{\dagger}\right)
$$

giving

$$
m_{H_{u}}^{2} \sim m_{H_{d}}^{2} \sim \frac{F_{X}^{2} M_{c}}{M_{*}^{3}}
$$

To summarize the results of this model, in the effective 4-dimensional theory at the massless level we have MSSM gauge fields and two Higgs doublets. At the compactification scale non-universal gaugino masses and the $\mu$ term are generated together with $B_{\mu}$ term and soft SUSY breaking Higgs masses. Soft SUSY breaking masses of squarks and sleptons and trilinear couplings are negligible at $M_{c}$. Furthermore, Yukawa couplings of $b$ and $\tau$ unify at $M_{c}$.

The fact that in some sectors of the theory there are GUT relations between parameters and in others there are no relations is clearly a consequence of having branes with grand unified gauge symmetry and with restricted gauge symmetry. The results of the above model can also be easily modified by different arrangements of fields living in the bulk and those localized on branes. For example if the third generation matter fields are not localized on the brane with GUT symmetry (they can be localized on the brane with restricted gauge symmetry or can come from different multiplets living in the bulk), the Yukawa unification is not predicted. ${ }^{4}$ Similarly if the Higgs fields do not live in the bulk and are not localized on the brane where SUSY is broken, the soft SUSY breaking Higgs masses are not generated. In this case the $\mu$ term is not generated either; however, it can be generated within the effective 4-dimensional theory 19]. Therefore, generating gaugino masses is not necessarily connected with either generating Higgs masses or Yukawa unification.

A very interesting situation can happen in models with $S O(10)$ GUT symmetry in higher dimensional spacetimes 10, 11]. There are more ways to break $S O(10)$ symmetry down to $G_{S M}$ and therefore branes on which the gauge symmetry is restricted to different subgroups of $S O(10)$ can appear in a model. Splitting of Higgs doublets and triplets can be achieved if the gauge symmetry on a brane is restricted to $S O(6) \times S O(4)$ which is isomorphic to the Pati-Salam gauge symmetry $S U(4) \times S U(2)_{L} \times S U(2)_{R}$. In a similar

\footnotetext{
${ }^{4}$ This can be actually very useful for treating first two generation, since the unification of Yukawa couplings of first two generation fermions is excluded by experimental observations.
} 
way as in Eqs. (2.1) - (2.3), masses of gauginos of $S O(6)$ and $S O(4)$ gauge groups can be generated on this brane separately [10],

$$
M_{6}=\frac{\lambda_{6}^{\prime} F_{X} M_{c}}{M_{*}^{2}}, \quad M_{4}=\frac{\lambda_{4}^{\prime} F_{X} M_{c}}{M_{*}^{2}} .
$$

The masses of gauginos of the MSSM $\left(M_{1}, M_{2}, M_{3}\right)$ are then given as

$$
M_{1}=\frac{2}{5} M_{6}+\frac{3}{5} M_{4}, \quad M_{2}=M_{4}, \quad M_{3}=M_{6} .
$$

The special form of $M_{1}$ is related to the way in which the generator of hypercharge is expressed as a linear combination of the generator of $B-L$ from $S O(6)$ and the generator of $t_{3 R}$ from $S O(4)$, namely $Y=\sqrt{\frac{2}{5}}(B-L)-\sqrt{\frac{3}{5}} t_{3 R}$ (for detailed discussion see [10]) ${ }^{5}$.

As in the case of $S U(5)$, Yukawa unification may or may not be present, depending on the way matter fields are introduced in the model. If the third generation matter fields are localized on a brane with $S O(10)$ symmetry, $t-b-\tau$ Yukawa unification is predicted. However, matter fields can be localized on a brane with $S U(5) \times U(1)$ gauge symmetry in which case only $b-\tau$ Yukawa unification should be expected at $M_{c}$. And of course models can be built without having any kind of Yukawa unification. Similarly soft SUSY breaking Higgs masses can be generated. If they are generated on the same brane as gaugino masses, $S O(6) \times S O(4)$ symmetry guarantees that $m_{H_{u}}^{2}=m_{H_{d}}^{2}$ at the compactification scale.

\section{Pati-Salam scenario}

In this section, we investigate the parameter space of gaugino mediated SUSY breaking models where gaugino masses are constrained by the Pati-Salam (PS) symmetry to obey

$$
M_{1}=\frac{3}{5} M_{2}+\frac{2}{5} M_{3}
$$

at the compactification scale. Scalar masses and trilinear soft SUSY breaking terms are restricted to be $m_{0} \simeq A_{0} \simeq 0$. In minimal gaugino mediation the bilinear $B_{0}$ parameter is also $\simeq 0$, which leads to a predicted value of $\tan \beta$. Here, we will work with a somewhat more general format, taking $\tan \beta$ as an independent parameter and determining the value of $B$ at the weak scale by imposing minimization conditions on the scalar potential, so that radiative EW symmetry breaking occurs (REWSB). Note that this procedure also determines the magnitude, but not the sign, of the superpotential Higgs mass term $\mu$.

Our goal is to delineate the allowed regions of the parameter space in this scenario, and to find the regions that can be explored by near future experiments. The parameter space of the model is then given by

$$
M_{2}\left(M_{c}\right), M_{3}\left(M_{c}\right), \tan \beta, \operatorname{sign}(\mu), M_{c}
$$

\footnotetext{
${ }^{5}$ Similarly, if gaugino masses are generated on a brane with flipped $S U(5)^{\prime} \times U(1)^{\prime}$ symmetry, we have relation $M_{2}=M_{3}=M_{5^{\prime}}$ and $M_{1}$ is a linear combination of $M_{5^{\prime}}$ and $M_{1^{\prime}}$. Therefore gaugino masses are given by two independent parameters $M_{1}$ and $M_{2}=M_{3}$.
} 
as the independent parameters. The sparticle spectrum will depend only logarithmically on the value of $M_{c}$, which nonetheless must be near the GUT scale. Hence, we adopt the choice $M_{c}=1 \times 10^{16} \mathrm{GeV}$, i.e. just below the usual unification scale of $M_{G U T} \simeq 2 \times 10^{16}$ $\mathrm{GeV}$.

We employ an updated version of ISAJET version 7.58[20] which allows for sparticle spectra predictions for negative values of the gaugino mass $M_{3}$ (as well as $M_{2}$ and $M_{1}$ as in earlier versions). Briefly, the subprogram ISASUGRA (which is a part of ISAJET) begins with weak scale values of gauge and Yukawa couplings, and evolves up in energy until the unification scale where $g_{1}=g_{2}$ is identified. At $M_{G U T}$ (or other intermediate scales), the soft SUSY breaking boundary conditions can be imposed, and evolution of the set of 26 coupled RGEs of the MSSM is calculated down to the weak scale, where the 1-loop effective potential is minimized at an optimized scale choice which reproduces to within $1-2 \mathrm{GeV}$ the light scalar Higgs mass $m_{h}$ as predicted by the FeynHiggsFast program[21]. Yukawa couplings are updated via their SUSY loop corrections, and the process of up-down RGE evolution is iterated until a stable solution is obtained, using the complete set of two-loop RGEs. A variety of non-universal soft SUSY breaking mass inputs are allowed, including for this study independent gaugino masses.

Our first results are presented in Fig. 1, where we show regions of the $M_{2}$ vs. $M_{3}$ parameter plane for $\mu<0$ and $\tan \beta=5,10,15,20,25$ and 30. The regions with low values of $\left|M_{i}\right|$ denoted by red crosses give rise to tachyonic sparticle masses. In these cases, the Yukawa coupling contribution to the RGEs for top and bottom squarks is dominant, and quickly drives these squared masses to negative values. The red shaded regions do not give rise to an appropriate radiative breakdown of EW symmetry. The magenta regions give rise to a charged LSP, the lightest stau. This is the well-known problem associated with models of minimal gaugino mediation. The light and dark blue shaded regions give rise to spectra in violation of LEP and LEP2 limits from sparticle searches. In the case of LEP bounds, the $Z$ boson has non-negligible decays to sparticles, while in the LEP2 case we require $m_{\widetilde{W}_{1}}>100 \mathrm{GeV}, m_{\tilde{\ell}_{1}}>92 \mathrm{GeV}, m_{\tilde{\mu}_{1}}>85 \mathrm{GeV}, m_{\tilde{\tau}_{1}}>68 \mathrm{GeV}$ and $m_{\widetilde{Z}_{1}}>37$ $\mathrm{GeV}$ [22]. The green shaded region obeys all constraints with the possible exception of LEP2 Higgs searches. These range from $m_{h}>114 \mathrm{GeV}$ for low $\tan \beta$ to $m_{h}>91 \mathrm{GeV}$ for $\tan \beta>10$ 23]. We show instead the black contour where $m_{h}=114 \mathrm{GeV}$, and where $m_{h}>114 \mathrm{GeV}$ occurs for larger values of $M_{2}$ and $M_{3}$.

For $\tan \beta=5$, a significant green region survives all constraints with the exception being that the light Higgs scalar has mass $m_{h}<114 \mathrm{GeV}$ except for a tiny region in the lower left. As $\tan \beta$ increases, the green allowed region gradually shrinks, while the tachyonic and stau LSP regions increase. This is reasonable in that as $\tan \beta$ increases, the $b$ and $\tau$ Yukawa couplings increase, and they contribute more strongly to driving scalar squared masses to small or tachyonic values. As $\tan \beta$ increases, however, the light Higgs mass $m_{h}$ also increases, giving rise to regions with viable supersymmetric spectra. This verifies the conjecture in Ref. [10] that the increased flexibility in the non-universal gaugino masses can cure the stau LSP problem inherent in models of minimal gaugino mediation without beyond the GUT scale running. However, we must note that the region of viable parameter space is rather limited, and in fact disappears for both high and low values of 


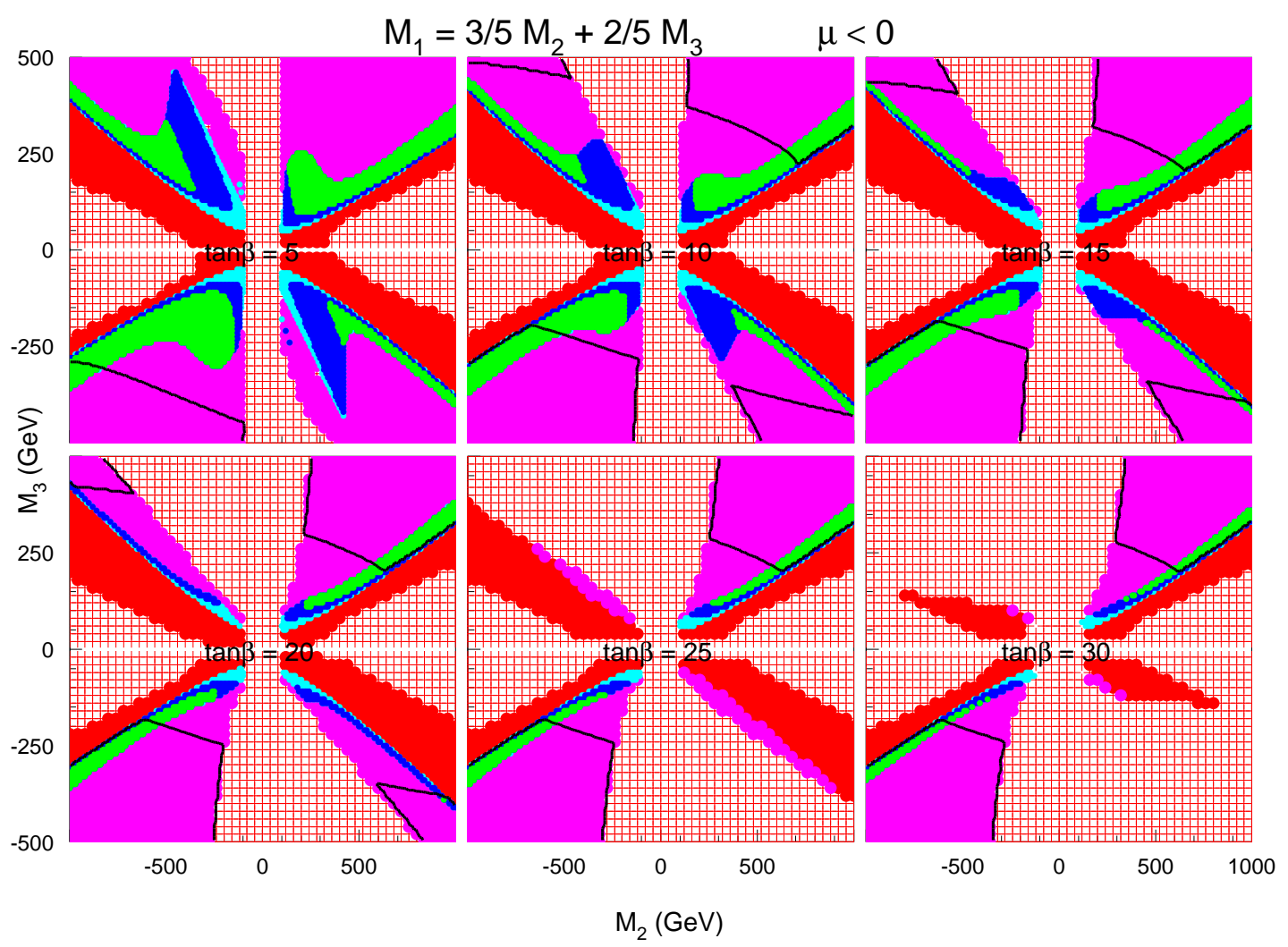

+ Tachyonic particles

- Ruled out by LEP2

- No EW symmetry breaking

- $\tilde{\chi}$ is not LSP

- $\mathrm{M}_{\mathrm{H}}=114 \mathrm{GeV}$

- Forbidden by $Z$ decay modes

- Allowed

Figure 1: Allowed and excluded regions of the $M_{2}\left(M_{c}\right)$ vs. $M_{3}\left(M_{c}\right)$ parameter plane in the PatiSalam scenario for various $\tan \beta$ values with $\mu<0$. Most of the parameter space is excluded by the lack of REWSB (red shaded), tachyonic particles in the spectrum (red crosses) or the stau being the LSP (magenta). Regions with viable spectra but in violation of limits from LEP (LEP2) are denoted by light blue (dark blue) shading. The black contour denotes regions where the light Higgs mass $m_{h}>114 \mathrm{GeV}$. Finally, the green shaded region is allowed by all constraints (except possibly those from LEP2 Higgs searches).

$\tan \beta$.

In our plots, both positive as well as negative gaugino mass parameters are allowed. In fact, we observe a near symmetry of parameter space when $M_{2} \rightarrow-M_{2}$ and $M_{3} \rightarrow-M_{3}$. In this case, $M_{1} \rightarrow-M_{1}$ as well, and all gaugino masses flip sign, but remain with the same absolute value. The MSSM Lagrangian is in fact invariant under the simultaneous 


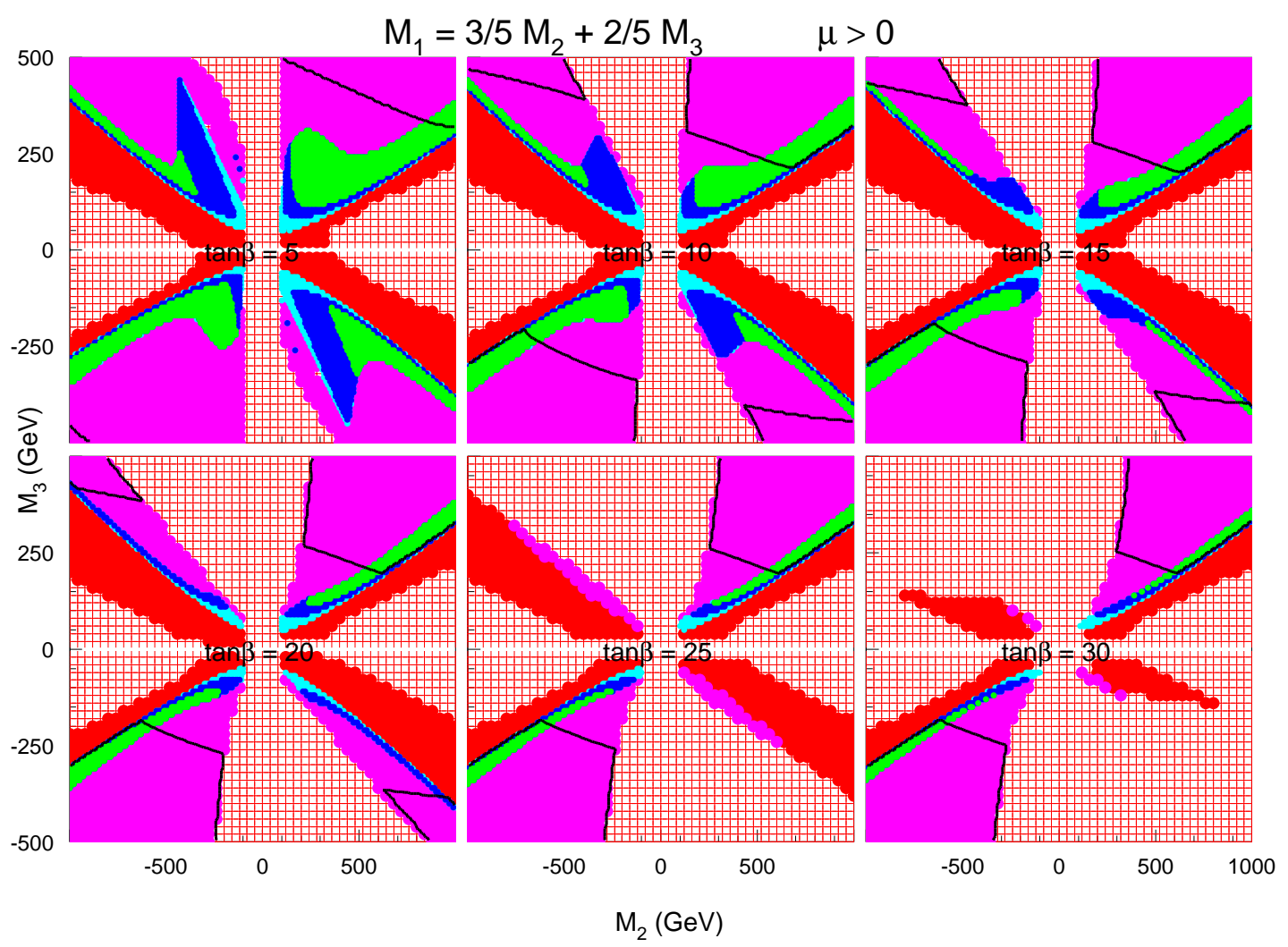

+ Tachyonic particles

- Ruled out by LEP2

- No EW symmetry breaking

- $\tilde{\chi}$ is not LSP

- $\mathrm{M}_{\mathrm{H}}=114 \mathrm{GeV}$

- Forbidden by $Z$ decay modes

- Allowed

Figure 2: Same as in Fig. 1, but with $\mu>0$.

sign change of the gaugino masses, the $A$ and $B$ parameters and $\mu,{ }^{6}$ so the near symmetry under sign flip of all gaugino masses may not be surprising. If instead we flip the sign of just one of $M_{2}$ or $M_{3}$, then of course there is much less symmetry, since a different absolute value of the bino mass $M_{1}$ is generated. Toward larger values of $\tan \beta$, this asymmetry increases because for $\left(M_{2} \cdot M_{3}\right)<0,\left|M_{1}\right|$ becomes smaller than in the $\left(M_{2} \cdot M_{3}\right)>0$ case, and the tau Yukawa coupling can more easily drive the right handed stau squared mass to negative values.

To illustrate the sort of spectra that can occur in the PS scenario, we show three cases in Table 1 for low, moderate and high $\tan \beta$. In the first case, universal gaugino masses are actually taken along the line where $M_{2}=M_{3}$. Along this line, however, the light Higgs

\footnotetext{
${ }^{6}$ We thank X. Tata for emphasizing this point.
} 
Table 1: Representative weak scale (physical) sparticle masses (in GeV units) and parameters for three Pati-Salam scenarios. We take $M_{c}=1 \times 10^{16} \mathrm{GeV}$.

\begin{tabular}{lccc}
\hline parameter & \multicolumn{3}{c}{ value } \\
\hline$M_{1}\left(M_{c}\right)$ & 250 & 590 & 666 \\
$M_{2}\left(M_{c}\right)$ & 250 & 800 & 900 \\
$M_{3}\left(M_{c}\right)$ & 250 & 275 & 315 \\
$\tan \beta$ & 5 & 10 & 30 \\
$\mu$ & $>0$ & $<0$ & $>0$ \\
$\mu\left(M_{\text {weak }}\right)$ & 337.6 & -153.5 & 147.0 \\
$B\left(M_{\text {weak }}\right)$ & 81.7 & -186.3 & 62.5 \\
$m_{\tilde{g}}$ & 583.9 & 652.3 & 738.9 \\
$m_{\tilde{u}_{L}}$ & 519.5 & 729.1 & 823.6 \\
$m_{\tilde{d}_{R}}$ & 502.8 & 541.6 & 614.0 \\
$m_{\tilde{t}_{1}}$ & 376.7 & 344.3 & 398.0 \\
$m_{\tilde{b}_{1}}$ & 485.2 & 536.3 & 565.5 \\
$m_{\tilde{e}_{L}}$ & 174.0 & 531.4 & 596.6 \\
$m_{\tilde{e}_{R}}$ & 101.4 & 222.1 & 249.6 \\
$m_{\tilde{\nu}_{e}}$ & 156.1 & 525.5 & 591.3 \\
$m_{\tilde{\tau}_{1}}$ & 98.4 & 215.4 & 162.6 \\
$m_{\tilde{\nu}_{\tau}}$ & 156.0 & 524.2 & 577.7 \\
$m_{\widetilde{Z}_{2}}$ & 172.6 & 161.2 & 152.6 \\
$m_{\widetilde{W}_{1}}$ & 171.3 & 153.2 & 144.7 \\
$m_{\widetilde{Z}_{1}}$ & 98.2 & 141.9 & 134.7 \\
$m_{h}$ & 109.3 & 115.2 & 118.0 \\
$m_{A}$ & 377.3 & 541.0 & 511.4 \\
$m_{H^{+}}$ & 385.6 & 547.2 & 519.1 \\
\hline & & &
\end{tabular}

mass $m_{h}$ is always below the limit from LEP2, and so is excluded. In addition, for the first case, the $\tilde{\tau}_{1}$ and $\widetilde{Z}_{1}$ are nearly mass degenerate. The spectra for the other two cases are taken from the upper right branches of allowable parameter space in Figs. 1 and 2.

Moving to regions beyond the black $m_{h}=114 \mathrm{GeV}$ contour, we find the allowed parameter space is bounded from below by regions where $\mu^{2}<0$ (no REWSB) and from above by where the $\tilde{\tau}_{1}$ becomes the LSP. These cases typically have small values of $|\mu|$, and hence a higgsino-like LSP. The $\widetilde{W}_{1}\left(\widetilde{Z}_{2}\right)$ will decay into three body modes dominated by virtual $W(Z)$ exchange. There is a very small mass gap between $\widetilde{W}_{1}$ and $\widetilde{Z}_{1}$, so that $\widetilde{W}_{1}$ and $\widetilde{Z}_{2}$ decay products will be quite soft, making collider searches challenging. One can expect that the search for lightest sleptons - selectron, smuon or stau - will be a better choice to hunt for SUSY particles.

In Fig. 3, we show in addition the relic density of neutralinos and the region excluded 


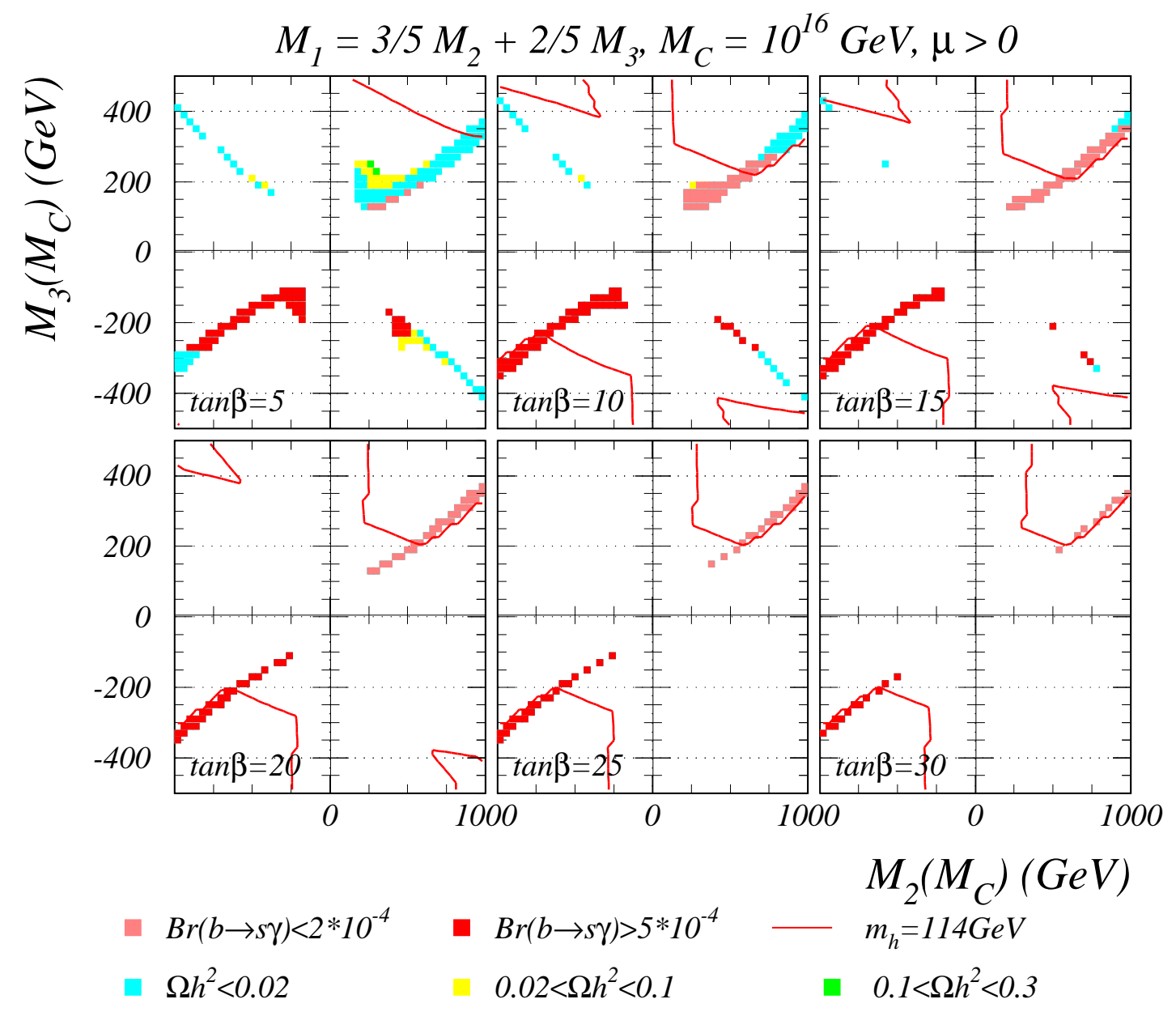

Figure 3: Same parameter space as in Fig. 2, but showing the neutralino relic density $\Omega h^{2}$ in the viable regions of parameter space.

by $B F(b \rightarrow s \gamma)$ constraints in the allowed parameter space regions from Fig. 2. For $B F(b \rightarrow s \gamma)$, we use the program of Ref. [24], upgraded to include running $b$-quark mass effects which are important at large $\tan \beta$. The red regions give too high a value of $B F(b \rightarrow$ $s \gamma)>5 \times 10^{-4}$, while pink regions give too low a value: $<2 \times 10^{-4}$. We also evaluate the muon anomalous magnetic moment $a_{\mu}$ following the program of Ref. [25]. All of the allowed region not excluded by $B F(b \rightarrow s \gamma)$ falls within the range $-10 \times 10^{-10}<a_{\mu}<60 \times 10^{-10}$. We caution the reader that the excluded regions we present here and later on due to the $B F(b \rightarrow s \gamma)$ and $a_{\mu}$ criteria should be interpreted as illustrative. The first reason for this is the theoretical uncertainty and the imminent new experimental analysis for $a_{\mu}$. Secondly, the results on $B F(b \rightarrow s \gamma)$ are quite model-dependent - the potential contributions from small off-diagonal SSB masses to $B F(b \rightarrow s \gamma)$ can easily work in both ways - they can easily increase or decrease the deviations of $B F(b \rightarrow s \gamma)$ from the SM value.

For the neutralino relic density, we use the recent calculation of Ref. [26], which 


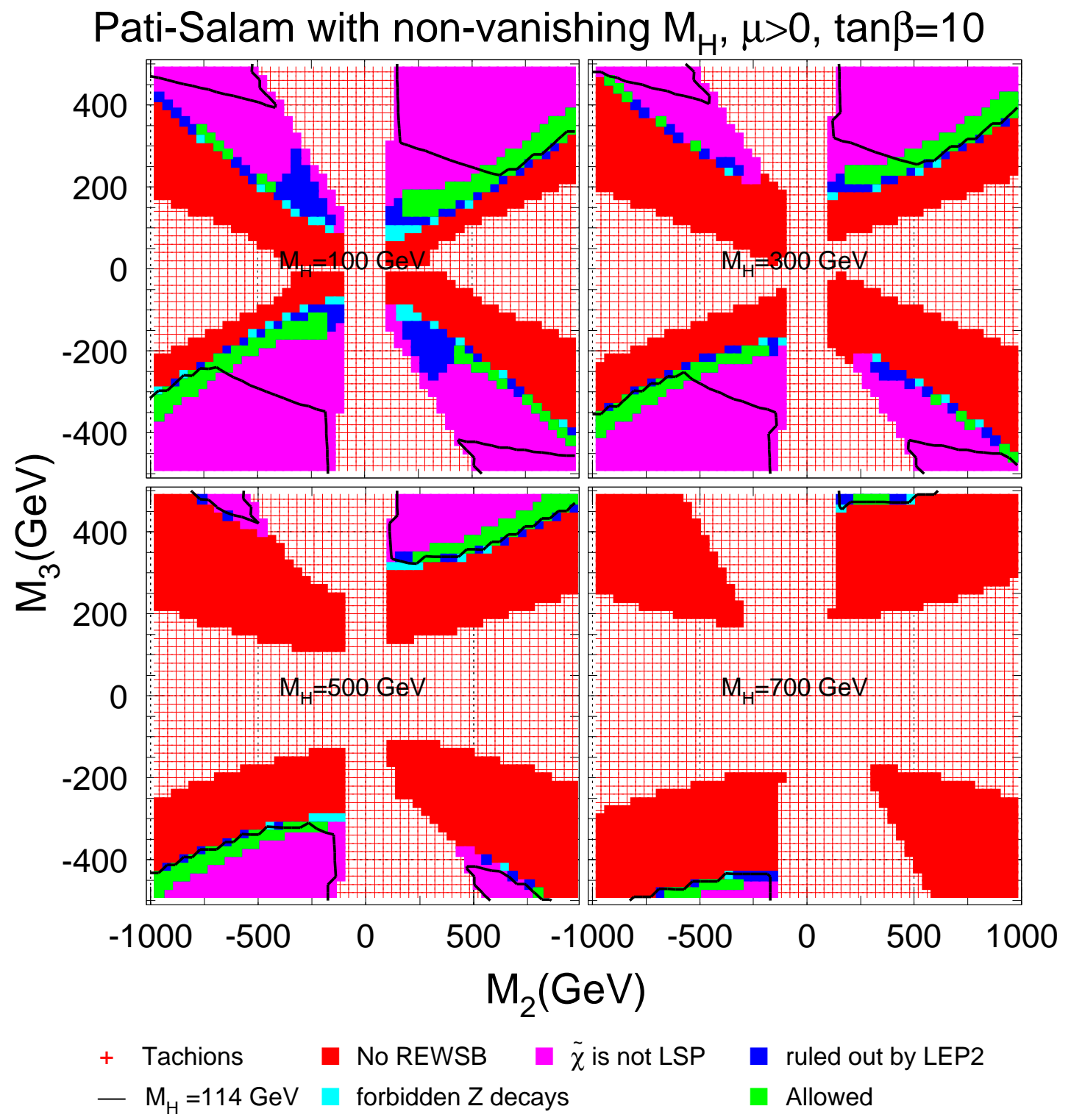

Figure 4: Allowed and excluded regions of the $M_{2}\left(M_{c}\right)$ vs. $M_{3}\left(M_{c}\right)$ parameter plane for the Pati-Salam scenario with $\mu>0$ and $\tan \beta=10$, but with $m_{H_{u}}\left(M_{c}\right)=m_{H_{d}}\left(M_{c}\right)=100,300500$ and $700 \mathrm{GeV}$. For $\mu<0$ the result is qualitatively the same.

includes all relevant co-annihilation channels as well as relativistic thermal averaging of annihilation cross sections times neutralino velocity. The blue regions have $\Omega h^{2}<0.02$, while yellow regions have $0.02<\Omega h^{2}<0.1$. The current cosmologically favored amount of CDM is $0.1<\Omega h^{2}<0.3$ (green). Thus, the neutralino relic density in the PS scenario is typically quite low compared with the cosmologically favored values of the CDM density. 


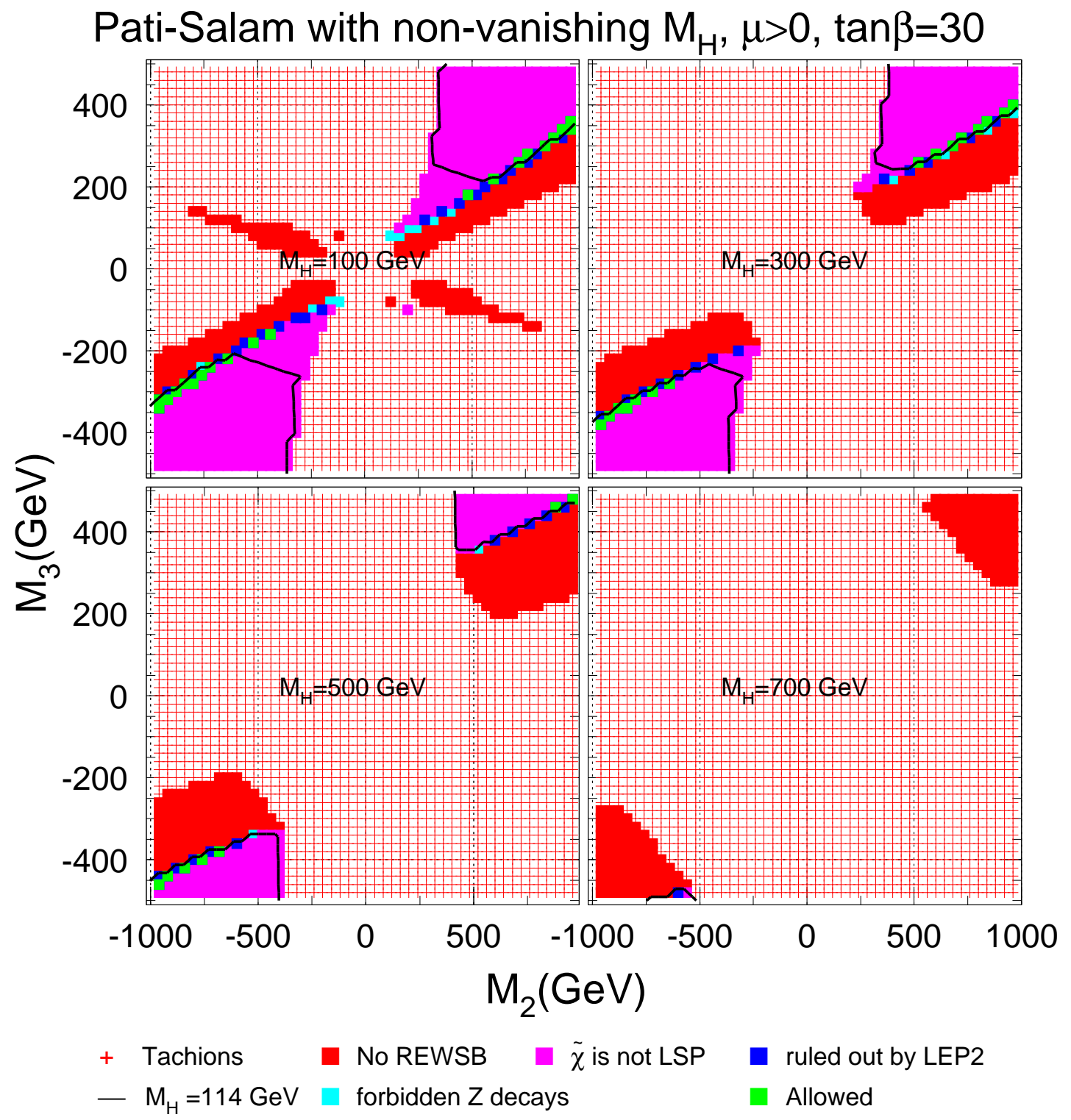

Figure 5: Allowed and excluded regions of the $M_{2}\left(M_{c}\right)$ vs. $M_{3}\left(M_{c}\right)$ parameter plane for the Pati-Salam scenario with $\mu>0$ and $\tan \beta=30$, but with $m_{H_{u}}\left(M_{c}\right)=m_{H_{d}}\left(M_{c}\right)=100,300500$ and $700 \mathrm{GeV}$. For $\mu<0$ the result is qualitatively the same.

This is easy to understand, since the allowed parameter space of the PS scenarios have an LSP with a large higgsino component. In this case, there is a large annihilation rate into $W W, Z Z$ and $Z h$ pairs, which results in a general reduction of the expected CDM density. Since our scenario is a higher dimensional model, the low neutralino relic density can easily be augmented by other forms of CDM related to extra dimensions. Thus, the predicted 
low neutralino relic density does not restrict the allowed parameter space in the PS case, and leaves room for other forms of CDM inherent in extra-dimensional models.

If the Higgs fields of an extra dimensional SUSY GUT propagate in the bulk, then they may gain non-negligible soft SUSY breaking masses as well. This additional freedom of nonzero Higgs squared masses at the compactification scale may allow additional parameter space to open up in the PS scenario. In Fig. Q, we plot the allowed parameter space in the $M_{2}$ vs. $M_{3}$ plane for $\tan \beta=10$, but this time allowing for non-zero soft SUSY breaking Higgs masses $m_{H_{u}}=m_{H_{d}}=100,300,500$ and $700 \mathrm{GeV}$. In fact as the Higgs masses increase, the allowed parameter space moves to higher absolute $M_{3}$ values, but also diminishes in area. The general diminution of parameter space occurs because in the RGEs for squared slepton masses, there exist terms where Higgs masses multiply Yukawa couplings. These contributions drive the soft SUSY breaking slepton masses to lower values than in the $m_{H_{u}}=m_{H_{d}}=0$ case, giving larger regions of parameter space with a stau LSP.

Since the diminution of soft breaking sfermion masses is due to RGE terms involving Yukawa couplings, we expect the effect to be amplified for large $\tan \beta$, where the $\tau$ Yukawa coupling gets large. In fact this is shown in Fig. 5, where we show the same results, but for $\tan \beta=30$. In this case, there is very little allowed parameter space for low values of Higgs masses, and no parameter space left on the frames shown for the very large values of $m_{H_{i}}(i=u, d)$.

\section{Gaugino mediation with independent gaugino masses}

A more general scenario occurs when all three gaugino masses are independent at the compactification scale, as in Eq. (2.3). In this case, we again scan the $M_{2}$ vs. $M_{3}$ plane, but this time for different independent fixed values of gaugino mass $M_{1}$. In Fig. 6, we show the parameter space for $\tan \beta=10, \mu>0$ and fixed values of $M_{1}=500,1000,1500$ and $2000 \mathrm{GeV}$. The shading of the different forbidden and allowed regions is the same as in Figs. 11 and 2. We see that for $M_{1}=500 \mathrm{GeV}$, for low values of $M_{3}$, the parameter space is forbidden either by tachyonic particles or no REWSB; for small values of $M_{2}$, additional parameter space is ruled out by constraints from LEP2 on the mass of the lightest chargino. As $M_{1}$ increases, the $U(1)_{Y}$ contribution to the running of the soft SUSY breaking squared mass $m_{\tilde{\tau}_{R}}^{2}$ increases, so that the region of parameter space excluded by a $\tilde{\tau}_{1}$ LSP shrinks. The allowed region of parameter space grows to become a major fraction of the $M_{2}$ vs. $M_{3}$ plane.

We also show the same $M_{2}$ vs. $M_{3}$ parameter space for fixed values of $M_{1}$ for larger $\tan \beta$ values of 30 and 45 in Figs. 7 and 8, respectively. As $\tan \beta$ increases, the $\tau$ Yukawa coupling also increases, which suppresses the stau masses, so that more of parameter space is disallowed by either the $\tilde{\tau}_{1}$ LSP or tachyonic constraints. However, in all cases, if $M_{1}$ is taken to be sufficiently large, then the gauge contribution to $\tilde{\tau}_{i}$ running wins out, and much of the parameter space with large gaugino masses becomes again allowed.

In Table 2, we show several representative cases from the allowed parameter space with independent gaugino masses. The first case has $M_{1}\left(M_{c}\right)=500 \mathrm{GeV}$, while $M_{2}\left(M_{c}\right)=150$ 


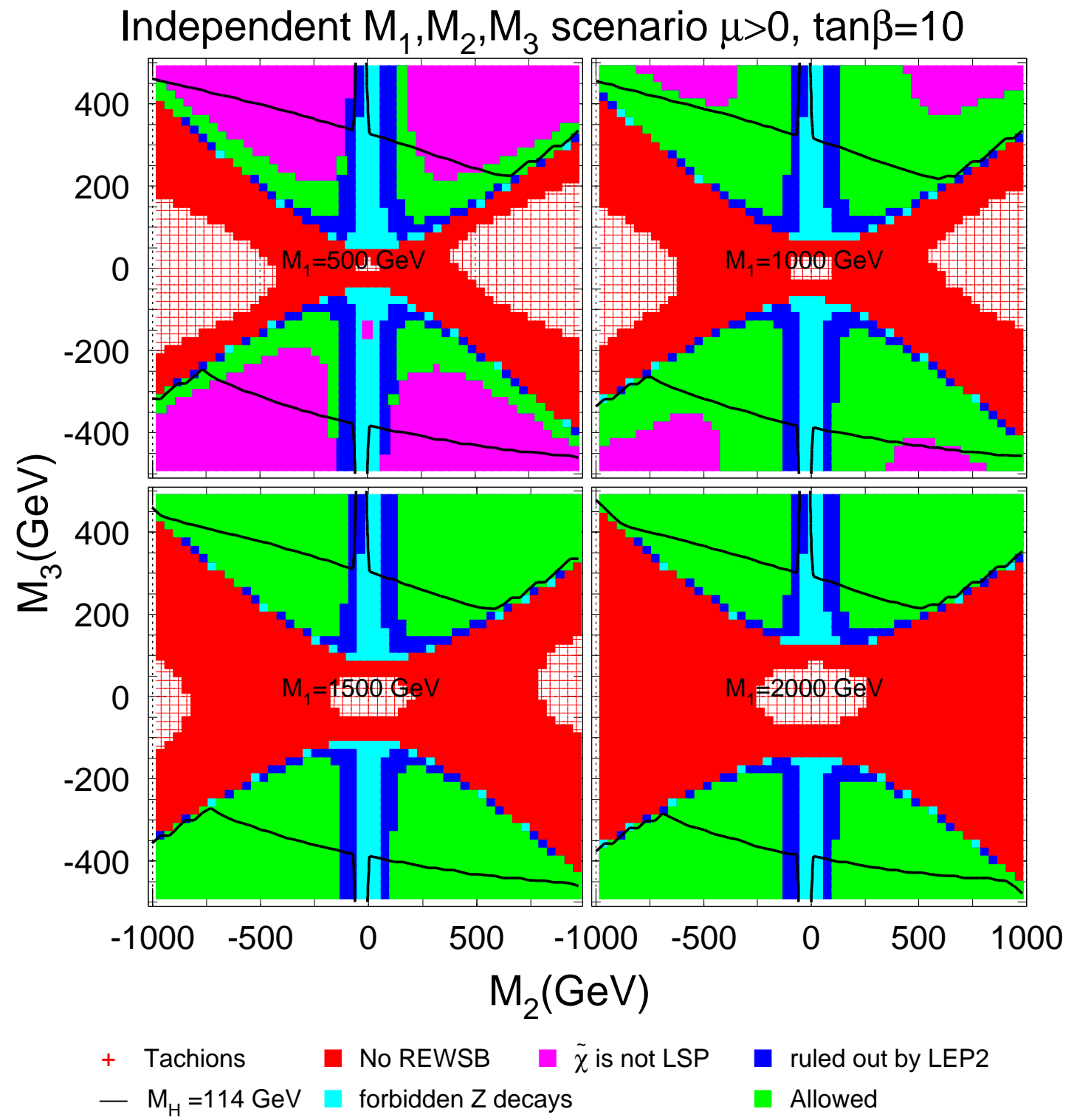

Figure 6: Allowed and excluded regions of the $M_{2}\left(M_{c}\right)$ vs. $M_{3}\left(M_{c}\right)$ parameter plane for independent gaugino masses at $M_{c}, \tan \beta=10$ and $\mu>0$. The four frames are shown for $M_{1}\left(M_{c}\right)=500$ 1000,1500 and $2000 \mathrm{GeV}$. For $\mu<0$ the result is qualitatively the same.

$\mathrm{GeV}$ and $M_{3}\left(M_{c}\right)=300 \mathrm{GeV}$. The most notable feature of this model is that $M_{2}\left(M_{\text {weak }}\right)=$ $111.9 \mathrm{GeV}$, compared with a $\mu$ value of $392.4 \mathrm{GeV}$. This results in the LSP being wino-like, and the $\widetilde{W}_{1}$ and $\widetilde{Z}_{1}$ being nearly mass degenerate. In fact, in this case the $\widetilde{W}_{1}$ is just beyond the kinematic reach of LEP2. However, its dominant decay mode is $\widetilde{W}_{1} \rightarrow \pi^{+} \widetilde{Z}_{1}$, and since the $\widetilde{W}_{1}-\widetilde{Z}_{1}$ mass gap is very small, the pion will be soft and not easily detected. 


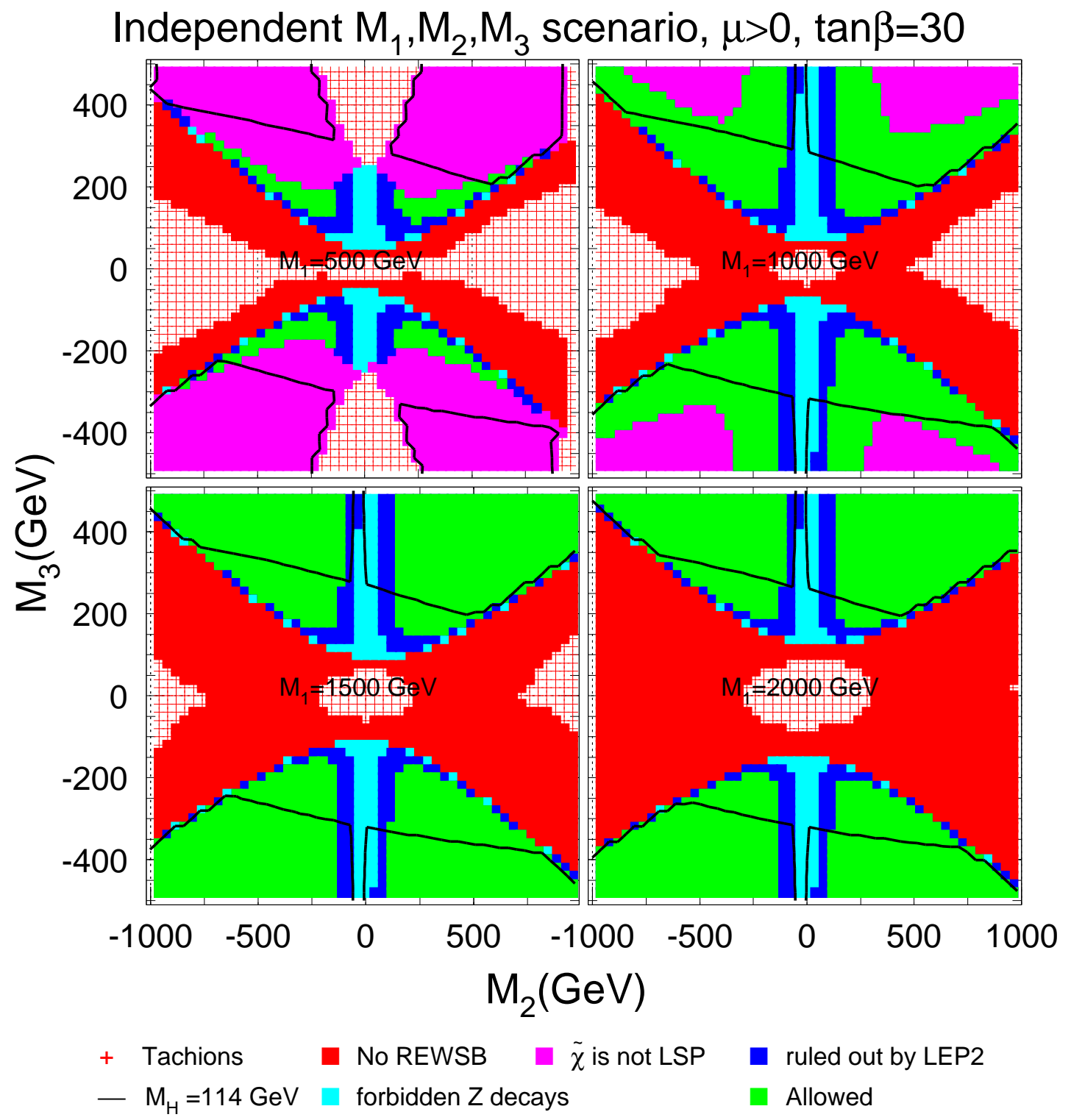

Figure 7: Allowed and excluded regions of the $M_{2}\left(M_{c}\right)$ vs. $M_{3}\left(M_{c}\right)$ parameter plane for independent gaugino masses at $M_{c}, \tan \beta=30$ and $\mu>0$. The four frames are shown for $M_{1}\left(M_{c}\right)=500$, 1000,1500 and $2000 \mathrm{GeV}$.

The chargino is sufficiently long-lived $(c \tau \simeq 0.1 \mathrm{~cm})$ that there may be a detectable track followed by kink in chargino production and decay. This is similar to the case from minimal anomaly-mediated SUSY breaking models (mAMSB) [2, 3], which also has a wino-like LSP. Other intriguing features of the spectra are that the light Higgs $h$ is just near the LEP2 bound, and that the sneutrinos are the lightest of all the sleptons, and that the left sleptons 


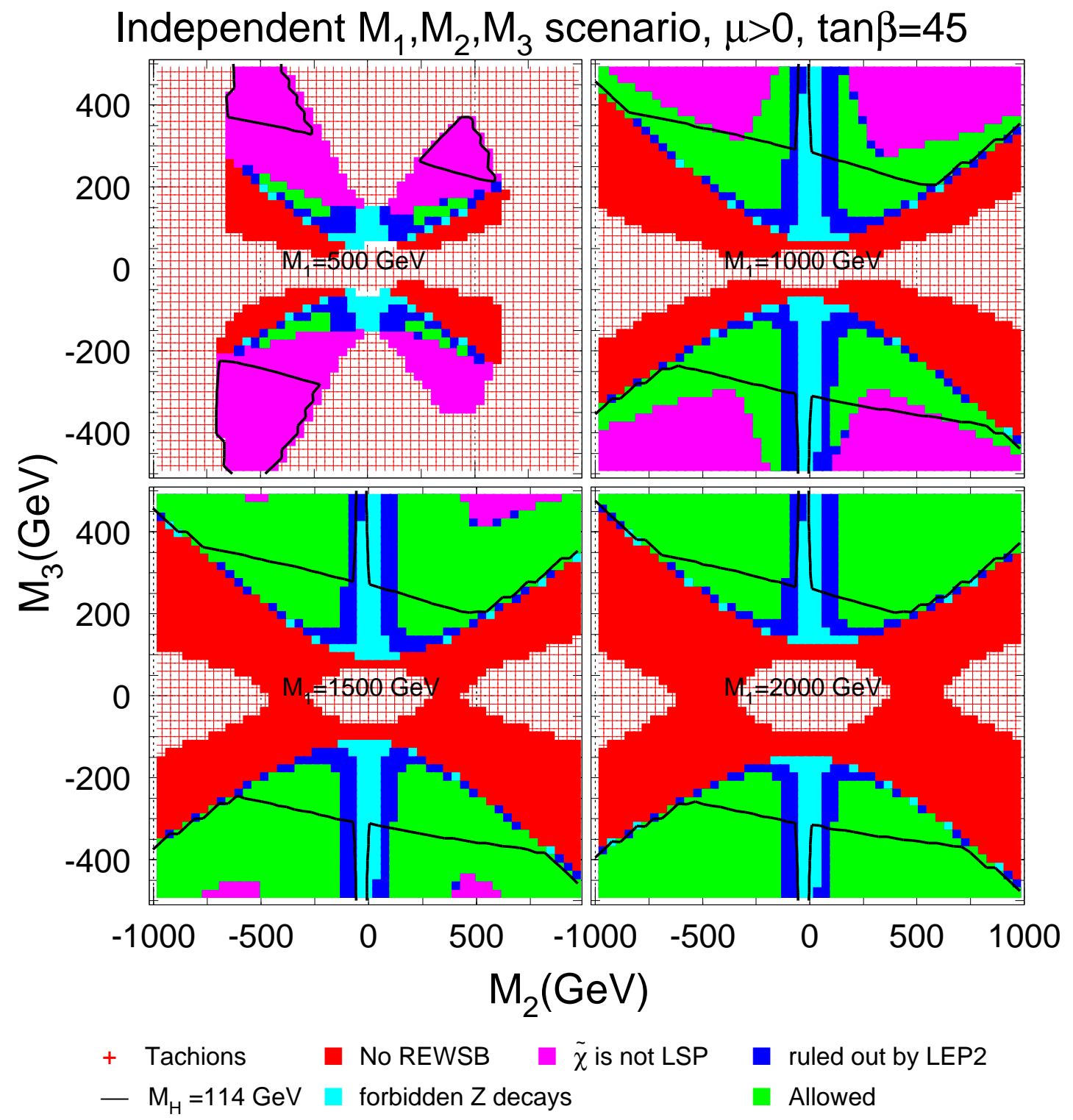

Figure 8: Allowed and excluded regions of the $M_{2}\left(M_{c}\right)$ vs. $M_{3}\left(M_{c}\right)$ parameter plane for independent gaugino masses at $M_{c}, \tan \beta=45$ and $\mu>0$. The four frames are shown for $M_{1}\left(M_{c}\right)=500$, 1000,1500 and $2000 \mathrm{GeV}$.

are far lighter than right sleptons, in contrast to the case in the minimal supergravity (mSUGRA) model.

The second case shown in Table 2 is quite different in that now $M_{1}$ and $\mu$ are comparable at the weak scale, and the LSP is a higgsino-bino mixture. There is a substantial mass gap between $\widetilde{W}_{1}$ and $\widetilde{Z}_{1}$, and the right- sleptons are lighter than left-sleptons. The 
Table 2: Representative weak scale (physical) sparticle masses (in GeV units) and parameters for three independent gaugino mass scenarios. We take $M_{c}=1 \times 10^{16} \mathrm{GeV}$.

\begin{tabular}{lccc}
\hline parameter & \multicolumn{3}{c}{ value } \\
\hline$M_{1}\left(M_{c}\right)$ & 500 & 500 & 1500 \\
$M_{2}\left(M_{c}\right)$ & 150 & 1000 & 500 \\
$M_{3}\left(M_{c}\right)$ & 300 & 350 & 400 \\
$\tan \beta$ & 10 & 10 & 30 \\
$\mu$ & $>0$ & $>0$ & $>0$ \\
$M_{2}\left(M_{\text {weak }}\right)$ & 112.0 & 796.1 & 393.2 \\
$\mu\left(M_{\text {weak }}\right)$ & 392.4 & 181.6 & 466.4 \\
$B\left(M_{\text {weak }}\right)$ & 42.1 & 244.4 & 22.1 \\
$m_{\tilde{g}}$ & 688.9 & 815.2 & 903.6 \\
$m_{\tilde{u}_{L}}$ & 595.0 & 909.6 & 824.2 \\
$m_{\tilde{d}_{R}}$ & 596.3 & 672.9 & 787.4 \\
$m_{\tilde{t}_{1}}$ & 471.6 & 424.5 & 645.7 \\
$m_{\tilde{b}_{1}}$ & 556.4 & 666.6 & 729.4 \\
$m_{\tilde{e}_{L}}$ & 139.1 & 653.2 & 425.2 \\
$m_{\tilde{e}_{R}}$ & 189.8 & 189.1 & 555.0 \\
$m_{\tilde{\nu}_{e}}$ & 114.4 & 648.4 & 417.7 \\
$m_{\tilde{\tau}_{1}}$ & 128.4 & 176.7 & 398.7 \\
$m_{\tilde{\nu}_{\tau}}$ & 113.6 & 646.9 & 398.2 \\
$m_{\widetilde{Z}_{2}}$ & 206.6 & 186.3 & 470.7 \\
$m_{\widetilde{W}_{1}}$ & 104.1 & 178.2 & 364.5 \\
$m_{\widetilde{Z}_{1}}$ & 103.7 & 156.2 & 362.9 \\
$m_{h}$ & 114.1 & 117.8 & 118.3 \\
$m_{A}$ & 404.8 & 665.9 & 546.9 \\
$m_{H+}$ & 412.7 & 671.1 & 554.1 \\
\hline & & &
\end{tabular}

$\tilde{\tau}_{1}$ is quite light, and in fact the decays of $\widetilde{W}_{1}$ and $\widetilde{Z}_{2}$ are dominantly into staus.

The third case in Table 2 is at large $M_{1}$ and large $\tan \beta$, and the LSP is again wino-like, as in case 1 . The lighter sleptons are dominantly left eigenstates.

In Fig. 9, we show the relic density of neutralinos, the $B F(b \rightarrow s \gamma)$ excluded region, and the region excluded by $a_{\mu}$ constraints, for two sample frames of the case with general gaugino masses. The first frame has $M_{1}=500 \mathrm{GeV}, \tan \beta=10$ and $\mu>0$. The color coding is similar to Fig. 3, except that now regions excluded by $a_{\mu}$ are also shown as light and dark gray regions. When a region is excluded by both $B F(b \rightarrow s \gamma)$ and $a_{\mu}$ it is colored red or pink. We note that the excluded regions are not symmetric in $B F(b \rightarrow s \gamma)$ under $M_{3} \rightarrow-M_{3}$, since the $A$-parameters evolve quite differently, giving rise to different stop masses and also $\mu$ parameter. 


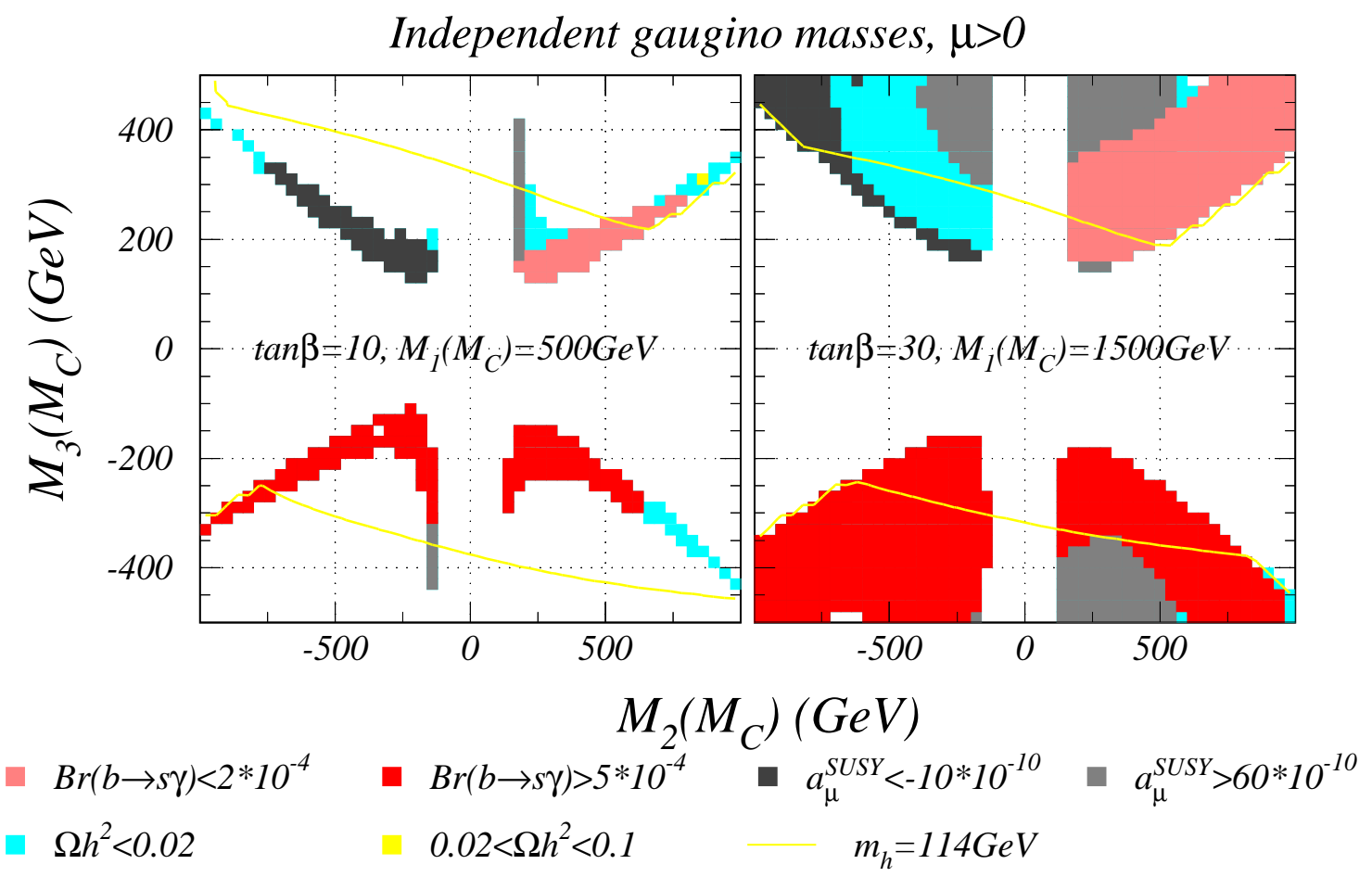

Figure 9: Regions of neutralino relic density in models with independent gaugino masses for $\tan \beta=10$ and $M_{1}=500 \mathrm{GeV}$, and for $\tan \beta=30$ and $M_{1}=1500 \mathrm{GeV}$. We take $\mu>0$.

Throughout almost all the allowed parameter space, the relic density $\Omega h^{2}$ is less than 0.02 (blue), i.e. not enough to explain the CDM needed by cosmological observations. This is not surprising in that over much of parameter space, the LSP is either wino-like or higgsino-like. In the first case, there is a large co-annihilation rate between $\widetilde{Z}_{1}$ and $\widetilde{W}_{1}$ that leads to a small relic density. In the second case, there is a large annihilation rate into $W W, Z Z$ and $Z h$ pairs, which also suppresses the relic density. There are a few points for large positive $M_{2}$ and $M_{3}$ where $\Omega h^{2}>0.02$. In this region, $\mu$ and $M_{2}$ are comparable at the weak scale, and the mass gap between $\widetilde{W}_{1}$ and $\widetilde{Z}_{1}$ can reach up to $20 \mathrm{GeV}$, thus suppressing the $\widetilde{W}_{1}-\widetilde{Z}_{1}$ coannihilation rate, and increasing the relic density. The second frame of Fig. 9 shows the relic density for $M_{1}=1500 \mathrm{GeV}, \tan \beta=30$ and $\mu>0$. In this case, the relic density is low over all the parameter space shown, due to the presence of a wino-like LSP. Again, a low neutralino relic density leaves room for other potential forms of CDM and does not restrict the allowed parameter space of the model.

\section{Conclusions}

Gaugino-mediated SUSY breaking is especially attractive in that it leads to a natural solution to the problem of flavor-changing and $C P$ violating processes that are generic to the MSSM. If the soft SUSY breaking boundary conditions from gaugino mediation apply at the GUT scale, then models with universal gaugino masses lead to a charged (stau) LSP. 
Additional running of soft SUSY breaking parameters above the GUT scale can lift the stau mass to the level where a neutralino LSP is possible. However, if the running occurs in a simple GUT, then the values of $\tan \beta$ consistent with Yukawa unification may conflict with the predicted value of $\tan \beta$ from minimal gaugino mediation with a small $B$ term.

In this paper we examined an alternative solution to the stau LSP problem, in that we allow non-universal gaugino masses. Extra dimensional GUT models can be easily constructed which include gaugino-mediated SUSY breaking, but non-universal gaugino masses. The additional freedom from the non-universal gaugino masses allows us to find viable regions of model parameter space where SUSY spectra are generated consistent with constraints on non-tachyonic sparticles, REWSB and constraints from LEP2. We examined the case of two independent gaugino masses, which occurs when SUSY breaking takes place on a hidden sector brane with Pati-Salam symmetry. In this case, limited regions of viable parameter space were found, usually with a higgsino-like LSP. Those regions vanish for the high values of $\tan \beta \sim 45-55$ where one could expect Yukawa coupling unification.

We also examined the case where all three gaugino masses were independent. In these models, choosing $M_{1}$ large enough opens up large regions of viable parameter space. In this case, the LSP might be either wino-like or higgsino-like. Regions with large $\tan \beta$ are allowed provided one chooses $M_{1}$ to be sufficiently large. We illustrated regions of parameter space constrained by muon $g-2$ and $B F(b \rightarrow s \gamma)$, although these constraints must be interpretted with caution. Due to the nature of the LSP, the relic density of neutralinos in these models is generally below expectations from cosmology; in this case, some other type of matter (axions or hidden sector states) would be needed to account for the bulk of CDM needed in the universe.

\section{Acknowledgments}

This research was supported in part by the U.S. Department of Energy under contract number DE-FG02-97ER41022. A.M. is supported in part by the National Science Foundation under Grant PHY-0071054, and by a Research Innovation Award from Research Corporation. R.D. thanks S. Raby and Y. Nomura for discussions. R.D. is supported in part by DOE grant DOE/ER/01545-830.

\section{References}

[1] M. Dine and A. E. Nelson, Phys. Rev. D 48 (1993) 1277;

M. Dine, A. E. Nelson and Y. Shirman, Phys. Rev. D 51 (1995) 1362;

M. Dine, A. E. Nelson, Y. Nir and Y. Shirman, Phys. Rev. D 53 (1996) 2658;

for a review, see G. F. Giudice and R. Rattazzi, Phys. Rev. 322 (1999) 419.

[2] L. Randall and R. Sundrum, Nucl. Phys. B 557 (1999) 79.

[3] G. F. Giudice, M. A. Luty, H. Murayama and R. Rattazzi, J. High Energy Phys. 12 (1998) 027 .

[4] D. E. Kaplan, G. D. Kribs and M. Schmaltz, Phys. Rev. D 62 (2000) 035010;

Z. Chacko, M. A. Luty, A. E. Nelson and E. Ponton, J. High Energy Phys. 0001 (2000) 003. 
[5] M. Schmaltz and W. Skiba, Phys. Rev. D 62 (2000) 095004; ibid Phys. Rev. D 62 (2000) 095005.

[6] H. Baer, M. Diaz, P. Quintana and X. Tata, J. High Energy Phys. 0004 (2000) 016; H. Baer, A. Belyaev, T. Krupovnickas and X. Tata, Phys. Rev. D 65 (2002) 075024.

[7] Y. Kawamura, Prog. Theor. Phys. 105 (2001) 999; ibid Prog. Theor. Phys. 105 (2001) 691.

[8] L. Hall and Y. Nomura, Phys. Rev. D 64 (2001) 055003.

[9] G. Altarelli and F. Feruglio, Phys. Lett. B 511 (2001) 257;

A.B. Kobakhidze, Phys. Lett. B 514 (2001) 131;

A. Hebecker and J. March-Russell, Nucl. Phys. B 613 (2001) 3.

[10] R. Dermíšek and A. Mafi, Phys. Rev. D 65 (2002) 055002.

[11] L. Hall, Y. Nomura, T. Okui and D. Smith, Phys. Rev. D 65 (2002) 035008;

T. Asaka, W. Buchmüller and L. Covi, Phys. Lett. B 523 (2001) 199.

[12] See for example, T. Goto and T. Nihei, Phys. Rev. D 59 (1999) 115009;

K. S. Babu, J. C. Pati and F. Wilczek, Nucl. Phys. B 566 (2000) 33;

R. Dermíšek, A. Mafi and S. Raby, Phys. Rev. D 63 (2001) 035001;

H. Murayama and A. Pierce, Phys. Rev. D 65 (2002) 055009.

[13] K. R. Dienes, E. Dudas, T. Gherghetta, Phys. Lett. B 536 (1998) 55;

ibid Nucl. Phys. B 537 (1999) 47.

[14] Some recent analyses include, H. Baer, M. Diaz, J. Ferrandis and X. Tata, Phys. Rev. D 61 (2000) 111701;

H. Baer and J. Ferrandis, Phys. Rev. Lett. 87 (2001) 211803;

T. Blažek, R. Dermíšek and S. Raby, Phys. Rev. Lett. 88 (2002) 111804 .

ibid hep-ph/0201081;

For discussion of Yukawa unification in models with non-universal gaugino masses, see

S. Komine and M. Yamaguchi, hep-ph/0110032;

U. Chattopadhyay and P. Nath, Phys. Rev. D 65 (2002) 075009.

[15] C. Csaki, J. Erlich, C. Grojean and G. D. Kribs, Phys. Rev. D 65 (2002) 015003;

H. C. Cheng, D. E. Kaplan, M. Schmaltz and W. Skiba, Phys. Lett. B 515 (2001) 395.

[16] A. B. Lahanas and D. V. Nanopoulos, Phys. Rept. 145 (1987) 1.

[17] J. R. Ellis, K. Enqvist, D. V. Nanopoulos and K. Tamvakis, Phys. Lett. B 155 (1985) 381.

[18] S. F. King and D. A. J. Rayner, Nucl. Phys. B 607 (2001) 77.

[19] G.F. Giudice and A. Masiero, Phys. Lett. B 206 (1988) 480;

For an $S O(10)$ model see also, A. Mafi and S. Raby, Phys. Rev. D 63 (2001) 055010.

[20] H. Baer, F. Paige, S. Protopopescu and X. Tata, hep-ph/0001086.

[21] S. Heinemeyer, W. Hollik and G. Weiglein, hep-ph/0002213.

[22] See e.g. R. Barate et al. [ALEPH Collaboration], Phys. Lett. B 499 (2001) 67.

[23] LEP Higgs Working Group Collaboration, hep-ex/0107030.

[24] H. Baer and M. Brhlik, Phys. Rev. D 55 (1997) 3201; H. Baer, M. Brhlik, D. Castano and X. Tata, Phys. Rev. D 58 (1998) 015007.

[25] H. Baer, C. Balázs, J. Ferrandis and X. Tata, Phys. Rev. D 64 (2001) 035004.

[26] H. Baer, C. Balázs and A. Belyaev, J. High Energy Phys. 0203 (2002) 042. 